\title{
Solving for the Orbital Elements of Binary Systems using MCMC Simulations.
}

\author{
Kyle Mede ${ }^{1}$ and Timothy D. Brandt ${ }^{2}$ \\ ${ }^{1}$ University of Tokyo, Room 1121, Dept. of Astronomy, Hongo 7-3-1, Bunkyo-ku, \\ Tokyo, 113-0033, Japan \\ email: kylemede@astron.s.u-tokyo.ac.jp \\ ${ }^{2}$ Princeton University, Room 17, Dept. of Astronomy, Peyton Hall, 4 Ivy Lane, Princeton, \\ NJ 08544-1001, USA \\ email: tbrandt@astro.princeton.edu
}

\begin{abstract}
Recent simulation and observational data have been used to investigate the ability of Kozai oscillations to explain the formation of "hot Jupiter" planetary systems. One of the first exoplanets discovered, $\tau$ Boo Ab, orbits a star with a binary companion, making it an excellent testbed for this scenario. We have written a three-dimensional Markov Chain Monte Carlo (MCMC) simulator to constrain the orbit of the distant stellar companion $\tau$ Boo B, and are currently deriving orbital parameters and confidence intervals. These orbital parameters will confirm or reject Kozai oscillations as a plausible formation mechanism for $\tau$ Boo Ab.
\end{abstract}

Keywords. techniques: radial velocities, high angular resolution, astrometry, objects: $\tau$ Boo

Over 800 extrasolar planets (or exoplanets) have been confirmed so far, with nearly 2500 more candidates awaiting ground-based follow-up. Thanks to the large number of known planets, we are beginning to understand how planetary systems form and how abundant solar-like systems are. The first formation models assumed that planets formed on approximately coplanar and circular orbits, as we observe in our solar system today. However, as more planets were observed, it became clear that many $(\sim 17 \%)$ are in close-in orbits, while others are eccentric or misaligned (Nagasawa \& Ida 2011). For these planets, new mechanisms were proposed to explain their existence. One leading mechanism is Kozai oscillations, in which a stellar mass companion exchanges energy and angular momentum with the planet, producing oscillations in the planet's eccentricity and inclination.

Kozai oscillations may explain the origin of the short-period exoplanet $\tau$ Boo Ab. In this scenario, the distant $(\sim 225 \mathrm{AU}) \sim 0.4 \mathrm{M}_{\odot}$ companion star $\tau$ Boo B would have excited $\tau$ Boo Ab to high eccentricity (and close passages to its host star, $\tau$ Boo A), where tidal dissipation could damp the planet onto its observed short-period ( $\sim 3.3$ day), nearly circular orbit (Brogi et al. 2012). With strongly constrained orbital parameters the equations of Takeda et al. (2005) can be applied to compare the period of the Kozai oscillations to the $\sim 2 \mathrm{Gyr}$ age of $\tau$ Boo A, allowing for investigation if they could have played a role in the planet's formation.

Three previous investigations of $\tau$ Boo B's orbit have estimated its period to be anywhere from 390 to 2000 years, and its eccentricity to be from 0.42 to 0.91 (Hale 1994, Popović \& Pavlović 1996, Roberts et al. 2011), see figure (1). Even with 163 years of observations, $\tau$ Boo B has traversed less than half of the shortest proposed orbit. Investigations of such long-period systems require careful analyses, preferably with explicit priors and well-characterized posterior probability distributions. We therefore re-analyze the $\tau$ Boo system using Markov Chain Monte Carlo (MCMC). 


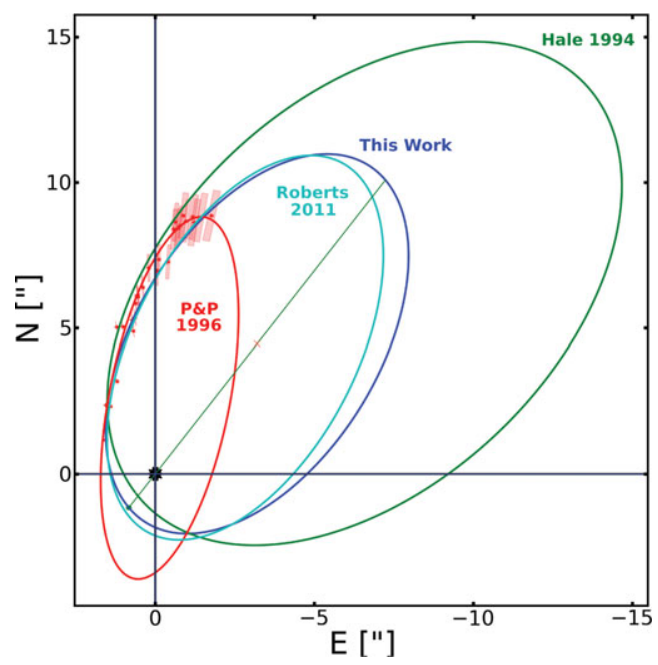

Figure 1. Three previously proposed orbits for $\tau$ Boo B (Hale 1994, P\&P 1996, and Roberts 2011), together with our best-fit. The locations of $\tau$ Boo B are shown as the small stars, with their associated errors, and $\tau$ Boo $\mathrm{A}$ at the origin. The proposed orbits have not converged to a consensus solution, which is needed for a complete assessment of the Kozai formation scenario.

One of the benefits of MCMC is that, in the limit of a long chain, its samples are drawn directly from the posterior probability distribution. A standard Monte Carlo simulation, by contrast, will draw many points in regions of parameter space with exceptionally low likelihood. MCMC can therefore be significantly more efficient at exploring a highdimensional parameter space in spite of the fact that its samples are not independent. Because MCMC directly probes the posterior probability distribution, calculating $68 \%$ and $95 \%$ confidence intervals for each parameter also becomes trivial.

Previous investigations of $\tau$ Boo B's orbit used only the positions measured from direct imaging data. However, radial velocity measurements of $\tau$ Boo A show a trend from $\tau$ Boo B superimposed on $\tau$ Boo Ab's 3.3 day orbit. We are taking advantage of these data, along with more recent astrometry, in a full three-dimensional simulator, which promises tighter orbital constraints and well-defined posterior probability distributions of the orbital parameters. Our initial results show a more eccentric orbit for $\tau$ Boo B than the most recent investigation, Roberts et al. (2011), with $e=0.79+/-0.01$.

Our new constraints on the orbit of $\tau$ Boo B will allow dynamical calculations of plausible orbital histories of the system over its 2 Gyr life, and will confirm or reject the possibility of a Kozai origin.

\section{References}

Brogi, M., Snellen, I. A. G., de Kok, R. J., Albrecht, S., Birkby, J., \& de Mooij,E. J. W. 2012, $A A P, 486, \mathrm{~L} 25$

Donati, J.-F., Moutou, C., Farès, R., Bohlender, D., Catala, C., Deleuil, M., Shkolnik, E., Collier Cameron, A., Jardine, M. M., \& Walker, G. A. H. 2008, MNRAS, 385, 1179

Hale, A. 1994, AJ, 107, 306

Popović, G. M. \& Pavlović, R. 1996, BAB, 153, 57

Nagasawa, M. \& Ida, S. 2011, ApJ, 742, 72

Roberts, Jr., L. C., Turner, N. H., ten Brummelaar, T. A., Mason, B. D., \& Hartkopf, W. I. $2011, A J, 142,175$

Takeda, G. \& Rasio, F. A. 2005, ApJ, 627, $1001 \mathrm{~T}$ 